\title{
Femur first surgical technique: a smart non- computer-based procedure to achieve the combined anteversion in primary total hip arthroplasty
}

Mattia Loppini ${ }^{1,2^{*}}$ D, Umile Giuseppe Longo ${ }^{3}$, Emanuele Caldarella', Antonello Della Rocca ${ }^{1}$, Vincenzo Denaro ${ }^{3}$ and Guido Grappiolo ${ }^{1}$

\begin{abstract}
Background: The relevance of prosthetic component orientation to prevent dislocation and impingement following total hip arthroplasty (THA) has been widely accepted. We investigated the use of a non-computer-based surgery to address the reciprocal orientation of the acetabular and femoral components.

Methods: In the femur first technique, the cup is positioned relative to the stem. When the definitive antetorsion of femoral component is fixed, the cup is positioned in a compliant anteversion to the stem. Clinical and radiographic assessments were performed before and 3 months after THA. Radiographic assessment was performed in standing position with the EOS 2D/3D radiography system. 3D images were used to preoperative anterior pelvic plane (APP) angle, postoperative acetabular inclination (Al) and anteversion (AA), and postoperative stem antetorsion. Clinical assessment was performed with Harris Hip Score (HHS).
\end{abstract}

Results: Forty patients (40 hips) underwent primary THA with an average age of 61 years (range, 36-84). Average HHS increased from $43 \pm 5$ (range, 37-52) preoperatively to $97 \pm 6$ (range, 86-100) at the last follow-up $(P<0.0001$ ). Average combined anteversion value of cup with liner and stem was $38^{\circ} \pm 9^{\circ}$ (range, $12^{\circ}-55^{\circ}$ ). Average Al value of cup with liner was $39^{\circ} \pm 6^{\circ}$ (range, $30^{\circ}-55^{\circ}$ ) in the group with standard stem and $45^{\circ} \pm 7^{\circ}$ (range, $39^{\circ}-58^{\circ}$ ) in the group with varized stem $(P=0.007)$. Relationship analysis showed no correlation between the combined anteversion values of the cup with liner and stem with APP angle values $(r=0.26, P=0.87)$.

Conclusions: Femur first technique allows the surgeon to achieve a combined anteversion ranging from $25^{\circ}$ to $50^{\circ}$ with a cup inclination ranging from $30^{\circ}$ to $50^{\circ}$. The cup is positioned according to the functional plane of the patient regardless the preoperative pelvic tilt.

Keywords: Combined anteversion, Acetabular inclination, Femur first, Hip, Arthroplasty

\footnotetext{
* Correspondence: mattia.loppini@gmail.com

${ }^{1}$ Hip Diseases and Joint Replacement Surgery Unit, Humanitas Clinical and

Research Centre, Via Alessandro Manzoni 56, 20089 Rozzano, Milan, Italy

${ }^{2}$ Humanitas University, Via Alessandro Manzoni 113, 20089 Rozzano, Milan,

Italy

Full list of author information is available at the end of the article
} 


\section{Background}

Over the last two decades, a growing relevance has been recognized to the prosthetic component orientation to prevent dislocation and impingement following total hip arthroplasty (THA).

In 1978, Lewinnek et al. [1] identified the orientation of the acetabular cup associated with the lower rate of dislocation. The suggested "safe zone" was included between $30^{\circ}-50^{\circ}$ of inclination in the coronal plane, and $5^{\circ}$ $25^{\circ}$ of anteversion in the axial plane. Although malpositioning of the acetabular component has been demonstrated to affect significantly the range of motion (ROM), joint stability, wear and loosening [2-5], the relative orientation of the acetabular and femoral components seems to be as important as the absolute positioning based on the bony landmarks.

The concept of combined anteversion was introduced by Ranawat et al. [6] in 1991, suggesting that the sum of the cup anteversion and stem antetorsion should be $45^{\circ}$ for women and between $20^{\circ}$ and $30^{\circ}$ for men. Subsequently, several mathematical models were developed to determine the combination of cup inclination and anteversion, and stem antetorsion providing the greater ROM and lower risk of cup-neck impingement [7-9]. Among these studies, the authors recommended a cup inclination between $40^{\circ}$ and $45^{\circ}$, combined with cup anteversion and stem antetorsion determined by the following formula: cup anteversion $+(0.7 \mathrm{x}$ stem antetorsion $)=\mathrm{X}^{\circ}$, where the value of $\mathrm{X}$ ranged from $37^{\circ}$ to $42^{\circ}$. On the other hand, clinical use of combined anteversion has determined it should be between $25^{\circ}$ up to $50^{\circ}$ [10].

Combined anteversion technique demonstrated to reduce of 6 times the dislocation rate in cementless total hip arthroplasty [11]. Some authors suggested the intraoperative navigation to achieve a proper combined anteversion [10, 12]. In this respect, computer-based systems can detect malpositioning of the first component, and correct accordingly the second component to restore the reciprocal orientation of both components. However, the setup and application of conventional navigation systems are time- and cost-consuming, and smart non-computer-based devices should be developed to allow the surgeon to achieve the proper combined anteversion [13].

In the present study, we investigated a non-computerbased surgical technique to obtain a proper orientation of both acetabular and femoral components during a primary THA procedure, according with the concept of combined anteversion. In the femur first technique, the cup is positioned relative to the stem after the trial stem has been implanted [14]. Therefore, the definitive antetorsion of femoral component is fixed, and the cup is positioned in a compliant anteversion to the first component. Moreover, because of a relation between cup inclination and the neck-to-shaft angle of straight stems has been described [15], we also hypothesized that the femur first technique could provide the coverage of the cup accordingly with the neck-to-shaft angle of the stem. Finally, because the present technique is based on the combined anteversion concept, we hypothesized that proper functional orientation of both cup and stem components should not be affected by the preoperative value of pelvic tilt.

Although previous studies evaluated the effectiveness of femur first technique to achieve a proper combined anteversion $[10,11,16]$, none investigated its role to determine the coverage of the cup, and the relationship of the combined anteversion values with pelvic tilt. The null hypotheses of the study were: 1) the femur first technique did not allow the surgeon to achieve a combined anteversion ranging from $25^{\circ}$ to $50^{\circ}$, and a cup inclination ranging from $30^{\circ}$ to $50^{\circ}$; and 2) there was no correlation between preoperative pelvic tilt and postoperative combined anteversion values if the femur first technique is performed.

\section{Methods \\ Sample}

Forty patients (40 hips) who underwent primary total hip arthroplasty from November 2014 to February 2015 were enrolled. Patients included 17 men and 23 women, with an average age of 61 years (range, 36-84) at the time of the index procedure. The average body mass index at the surgery was $27 \mathrm{~kg} / \mathrm{cm}^{2}$ (range, 18-39). The preoperative diagnosis included: primary osteoarthritis in 33 patients, osteoarthritis secondary to mild development dysplasia of the hip in 6 patients, and post-trauma osteoarthritis in one patient. Exclusion criteria included: patients eligible for partial or total THA revision, THA associated with other procedures (i.e. femoral osteotomy), previous pelvic and/or femoral osteotomy, severe hip dysplasia (Crowe III or IV), primitive or metastatic tumors of hip joint, previous spine and/or sacroiliac joint instrumentation, previous or current hip joint infection.

A minimum follow-up of 1 year was achieved in all patients.

\section{Surgical procedure}

In all patients, the digital preoperative radiograph in AP view was used to perform preoperative planning with the software Hip Arthroplasty Templating 2.4.3 running with OsiriX v.5.8.1 64-bit [17].

Surgery was performed by one experienced surgeon with the patient in the lateral position, through a posterolateral approach in all cases. After the release of short external rotators tendons at the insertion to greater trochanter, the capsule was incised, and the femur was dislocated. After the neck osteotomy, the femur was prepared by holding the knee flexed with the tibia in a 
vertical position. The starter device was introduced with the planned version for the definitive stem. The femoral rasps were inserted gradually increasing the size until proper fit was achieved. The definitive antetorsion of the stem was related with the native anatomy of the proximal femur. The type of neck, standard or varized, was defined according with the native value of caput-collum-diaphyseal angle (CCD), as measured with the radiographic assessment.

Maintaining the proper sized rasp in situ to protect the femoral shaft, the acetabulum was exposed, the labrum was removed, and the acetabulum was sequentially reamed. The definitive position of the acetabular component, in terms of anteversion and inclination angle, was identified basing of femoral stem anteversion and CCD angle. The femur was reduced with the proper sized rasp in situ by using a $44 \mathrm{~mm}$ trial plastic head with a long neck to compensate the absence of the acetabular component. By using a large head, it was possible to avoid an eccentric position of the head in the native acetabulum. After reduction, with the hip in neutral position at $0^{\circ}$ of flexion and abduction, the femur was internally rotated to obtain an angle of $35^{\circ}$, measured with a sterile goniometer, between the longitudinal axis of the tibia and the operating table which is parallel with the floor. Because of the degree of internal rotation to produce a coplanar head and cup is the combined anteversion [6], the definitive cup should be placed parallel with the horizontal line on the trial head in both axial and coronal plane. The parallelism in the axial plane provided the proper degree of cup anteversion to obtain a combined anteversion of $35^{\circ}$. On the other hand, the parallelism in the coronal plane provided the proper degree of cup inclination angle. The peripheral rim of the determined position of the cup was marked with a dermographic pen on the bony surface of the acetabulum. After the femur dislocation, the definitive acetabular cup was implanted, and osteophytes surrounding the acetabulum were removed when indicated. Then, the femur was reduced with the trial components in situ without the liner, and it was placed in internal rotation of $35^{\circ}$ with the hip in neutral position at $0^{\circ}$ of flexion and abduction. Therefore, the coplanarity of head and cup in both axial and coronal plane was checked, and the position of the liner with the elevated rim was determined in order to optimize the joint congruency. Previous joint dislocation, the femoral rasp was removed, the definitive liner was implanted with the elevated rim in planned position, and the definitive stem was implanted. Finally, the proper length of the neck was chosen to restore the native vertical and lateral offsets. At the end, the internal rotation with hip flexion and the free ROM were performed to assess the stability of the hip [18]. No impingement, dislocation and lower limb discrepancy were observed in any patient.
In all patients, uncemented cup and stem were implanted. The acetabular system was: G7 cup (Zimmer Biomet) in 31 hips and Trabecular Metal Acetabular Shell (Zimmer Biomet) in 9. The stem system was: GTS stem (Zimmer Biomet) in 23 hips and CLS stem (Zimmer Biomet) in 17. The stem was standard in 29 hips (21 GTS with CCD of $136^{\circ}$ and 8 CLS with CCD of $\left.135^{\circ}\right)$ and varized in 11 (2 GTS with CCD of $123^{\circ}$ and 9 CLS with CCD of $125^{\circ}$ ).

In all patients, the bearing surface consisted in ceramic head and polyethylene liner with $10^{\circ}$ elevated rim. The location of the elevated rim of the liner was reported by using a clock-face description where 12 o'clock indicated the side towards the head of patient, and 6 o'clock was the side towards the obturator foramen [17]. To clarify the data presentation, the site of the elevated rim was standardized to the right hip. The location of the elevated rim of the liner was: 12 o'clock in 1,11 o'clock in 12, 9 o'clock in 6, 7 o'clock in 17, and 3 o'clock in 4 hips.

\section{Clinical assessment}

The Harris Hip Score (HHS) was performed to evaluate the clinical picture of the patient before and 3 months after surgery. The questionnaire assigns up to 91 points for the domains of pain and hip function, and up to 9 points for the domain of range of motion. The final score ranges from 0 to 100 points, with the higher scores indicating the better clinical picture. The HHS has been considered excellent for values between 90 and 100 points, good for values between 80 and 89 , fair for values between 70 and 79 , and poor for values under 70 [19].

The intraoperative and postoperative complications, and the occurrence of hip dislocation were also evaluated.

\section{Radiographic assessment}

All patients had a radiographic assessment in standing position before and 3 months after THA with the EOS 2D/3D radiography system (Biospace Med, Paris, France).

EOS system allows to achieve an AP and lateral radiographic view of the whole skeletal system [20-22]. The 2D images were used to perform a 3D reconstruction of skeletal system and prosthetic components with a dedicated software (sterEOS 3D, versione 1.5.3.7947, Biospace Med, Paris, France). 3D images were used to measure preoperative CCD angle and femoral antetorsion [23], preoperative anterior pelvic plane (APP) angle, postoperative acetabular inclination (AI) and anteversion (AA), and postoperative stem antetorsion [24].

The CCD angle was defined as the angle between the femoral neck axis and femoral long axis [25]. The APP angle was defined as the angle subtented by a vertical reference line and a line tangent to the anterosuperior iliac spines and the pubic symphysis [22]. The combined anteversion was determined by the following formula: 
cup anteversion $+(0.7 \mathrm{x}$ stem antetorsion $)[8]$. The AI and AA were measured in the patient frame based on a vertical plane passing through the centre of the acetabular cup, which avoids the effect of a potential axial rotation of the pelvis during acquisition. On the other hand, the stem antetorsion was measured relative to posterior bi-condylar plane.

The AI and AA values measured with EOS system were also adjusted according the location of the liner's elevated rim. The 12 o'clock position was considered to reduce the $\mathrm{AI}$ value of $10^{\circ}$. The 3 o'clock and 9 o'clock positions were considered to reduce and increase the AA value of $10^{\circ}$, respectively. Finally, the 11 o'clock and 7 o'clock positions were not considered able to influence $\mathrm{AI}$ and AA values as measured with EOS system.

The outliers were the values out of the targeted ranges of combined anteversion (from $25^{\circ}$ to $50^{\circ}$ ) and AI (from $30^{\circ}$ to $50^{\circ}$ ).

\section{Statistics}

All the analyses were performed using SPSS for Mac (version 23.0, SPSS Inc., Chicago, Illinois). Descriptive statistics were calculated. The categorical variables were expressed as frequency with percentage. Continuous variable data were expressed as mean with standard deviation and range as minimum and maximum values.

The Wilcoxon signed-rank test with two tails was used to compare the preoperative and postoperative values of HHS. It was also used to compare the preoperative femoral antetorsion and postoperative stem antetorsion. The U Mann-Whitney test with two tails was used to compare the AI and combined anteversion values measured without liner and values recorded taking account the location of elevated rim of the liner. It was also used to compare the AI values of cup with liner in the group with standard stem and those in the group with varized stem. Finally, it was used to compare the combined anteversion values of the cup with liner and stem in the group with antetorsion of the stem $\leq 9^{\circ}$ and those in the group with $\geq 10^{\circ}$ of antetorsion of the stem.

The Spearman rank correlation matrix was used to assess the relationship between the combined anteversion values of the cup with liner and stem with APP angle values. For this relationship, a linear regression model was also performed and the coefficient of determination was calculated $\left(R^{2}\right)$. The $P$ was considered significant for values less than 0.05 .

\section{Results}

\section{Clinical outcome}

The average HHS increased from $43 \pm 5$ (range, 37-52) preoperatively to $97 \pm 6$ (range, 86-100) at the last follow-up $(P<0.0001)$. The final scores were good in 13 , and excellent in 27.
No patients reported intraoperative or postoperative complications neither dislocation of the prosthesis at the last follow-up.

\section{Radiographic outcome}

The average combined anteversion value with and without taking account the location of elevated rim of the liner was $38^{\circ} \pm 9^{\circ}$ (range, $12^{\circ}-55^{\circ}$ ) and $37^{\circ} \pm 13^{\circ}$ (range, $\left.2^{\circ}-65^{\circ}\right)$ respectively $(P=0.88)$. In the cup with liner group, there were $3(7.5 \%)$ outliers up to $13^{\circ}$, whereas there were $11(27.5 \%)$ outliers up to $23^{\circ}$ in the cup without liner group (Fig. 1).

The average AI value with and without taking account the location of elevated rim of the liner was $40^{\circ} \pm 6^{\circ}$ $\left(\right.$ range, $30^{\circ}-58^{\circ}$ ) and $41^{\circ} \pm 7^{\circ}$ (range, $30^{\circ}-58^{\circ}$ ) respectively $(P=0.8)$. In both groups, there were $3(7.5 \%)$ outliers up to $8^{\circ}$. In the subgroup analysis, the average AI value of cup with liner was $39^{\circ} \pm 6^{\circ}$ (range, $30^{\circ}-55^{\circ}$ ) in the group with standard stem and $45^{\circ} \pm 7^{\circ}$ (range, $39^{\circ}-58^{\circ}$ ) in the group with varized stem $(P=0.007)$ (Fig. 2$)$.

The average preoperative femoral antetorsion was $11^{\circ} \pm 14^{\circ}$ (range, $21^{\circ}$ of retrotorsion $-45^{\circ}$ of antetorsion), whereas the average postoperative stem antetorsion was $9^{\circ} \pm 10^{\circ}$ (range, $23^{\circ}$ of retrotorsion $-31^{\circ}$ of antetorsion) $(P=0.71)$. The average combined anteversion value of the cup with liner and stem was $34^{\circ} \pm 8^{\circ}$ (range, $23^{\circ}-51^{\circ}$ ) in the group with antetorsion of the stem $\leq 9^{\circ}$ and $41^{\circ} \pm 9^{\circ}\left(\right.$ range, $\left.12^{\circ}-55^{\circ}\right)$ in the group with $\geq 10^{\circ}$ of antetorsion of the stem $(P=0.002)$ (Fig. 3). Thirteen out of $19(68 \%)$ patients with a preoperative femoral antetorsion $\geq 10^{\circ}$ reported a postoperative antetorsion of the stem $\geq 10^{\circ}$ as well.

The relationship analysis showed no correlation between the combined anteversion values of the cup with liner and stem with APP angle values $(r=0.26, P=0.87)$ and no linear regression between these variables $\left(R^{2}=0.004\right.$, $P=0.71$ ) (Fig. 4).

\section{Discussion}

In the present study, we rejected the first null hypothesis and we accepted the second null hypothesis: 1 ) the femur first technique allowed to achieve a combined anteversion ranging from $25^{\circ}$ to $50^{\circ}$ with a cup inclination ranging from $30^{\circ}$ to $50^{\circ}$; and 2) there was no correlation between preoperative pelvic tilt and postoperative combined anteversion values.

About the first hypothesis, the combined anteversion value was within the targeted range in $92.5 \%$ of hips with an average value of $38^{\circ} \pm 9^{\circ}$. The results achieved with our non-computer-based procedure were similar to those reported by Dorr et al. [10] with intraoperative computer navigation. The authors found a combined anteversion within the targeted range from $25^{\circ}$ to $50^{\circ}$ in $96 \%$ hips with an average value of $35.9^{\circ} \pm 6.7^{\circ}$. On the 


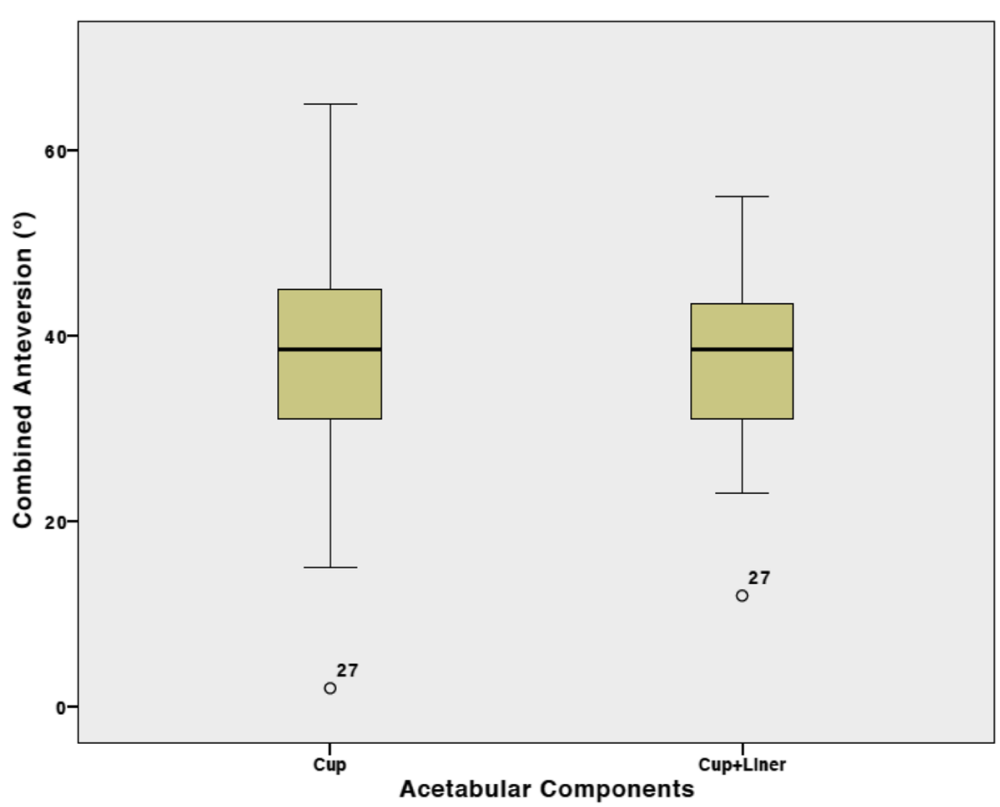

Fig. 1 Box plots showing the combined anteversion values measured in the cup with and without liner groups. Angles are expressed in degrees

other hand, Nakashima et al. [11] reported with their manual technique a combined anteversion within the targeted range from $40^{\circ}$ to $60^{\circ}$ in $73 \%$ hips with an average value of $50.3 \pm 6.2^{\circ}$. Although the manual placement of the cup was associated with $27 \%$ of outliers, the combined anteversion technique significantly reduced the dislocation after primary THA.

In our current practice, we prefer to use a liner with $10^{\circ}$ elevated rim if polyethylene is implanted to increase the hip stability $[26,27]$. Therefore, we would suggest to use the elevated rim to improve the joint congruency even if the combined anteversion is within the targeted range. In our series, in 29 (72.5\%) hips, the elevated rim was not used to change the combined anteversion and it was placed in the posterosuperior quadrant (11 o'clock) or posteroinferior quadrant (7 o'clock) to further increase the hip stability in internal rotation or flexion of the femur respectively. In the remaining hips $(27.5 \%)$ we used the elevated rim to improve the combined anteversion as required by the intraoperative assessment with

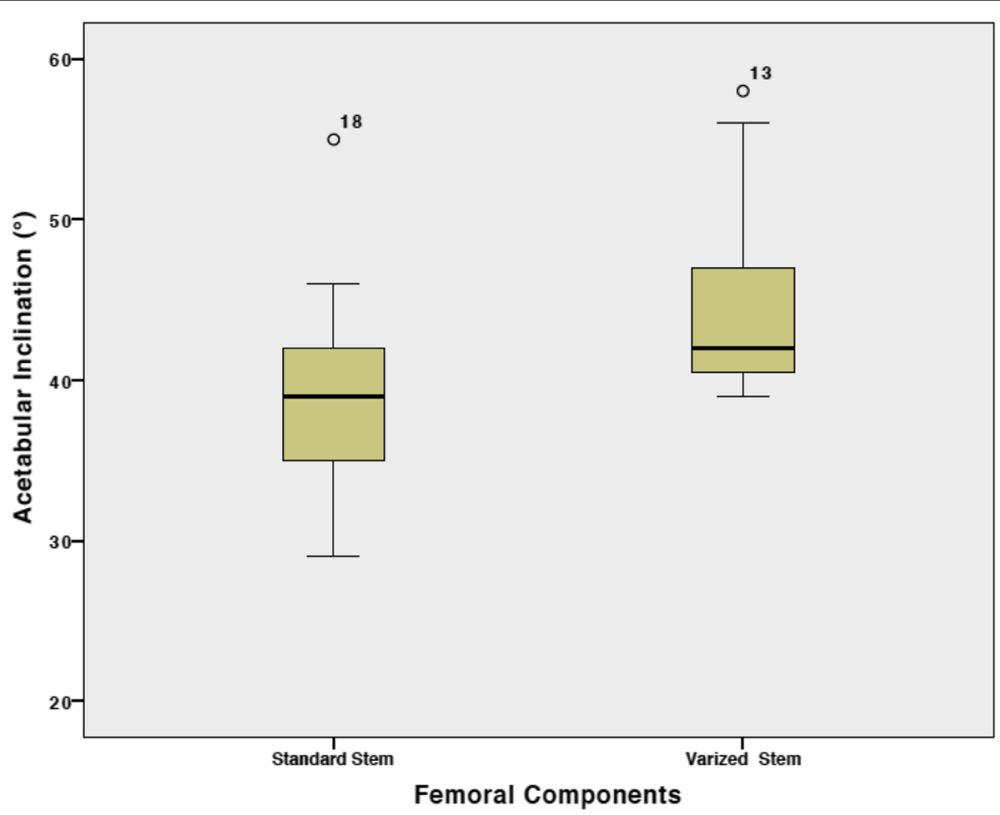

Fig. 2 Box plots showing the acetabular inclination values measured in the standard stem and varized stem groups. Angles are expressed in degrees 


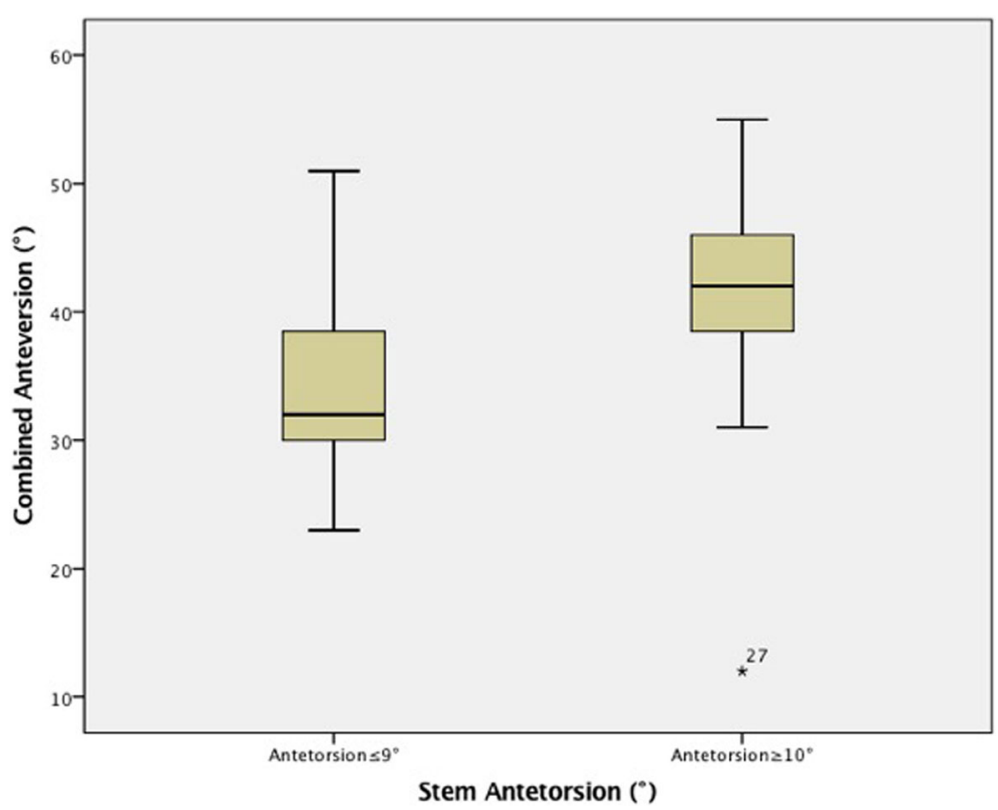

Fig. 3 Box plots showing the combined anteversion values measured with the cup-liner system in the group with $\leq 9^{\circ}$ and $\geq 10^{\circ}$ of antetorsion of the stem. Angles are expressed in degrees

the femur at $35^{\circ}$ of internal rotation. Because there was no impingement or hip subluxation or dislocation, the position of the cup was not changed in these patients, but the combined anteversion was improved by positioning the elevated rim anteriorly (3 o'clock) or posteriorly (9 o'clock).

The analysis of the combined anteversion values according with the antetorsion values of the stem showed that the combined anteversion values in the group with $\geq 10^{\circ}$ of stem antetorsion were significantly higher than those in the group with stem antetorsion $\leq 9^{\circ}(P=0.002)$. These findings demonstrated that the combined anteversion is strongly affected by the femoral antetorsion suggesting morphotype plays a critical role in the position of the definitive prosthetic components.

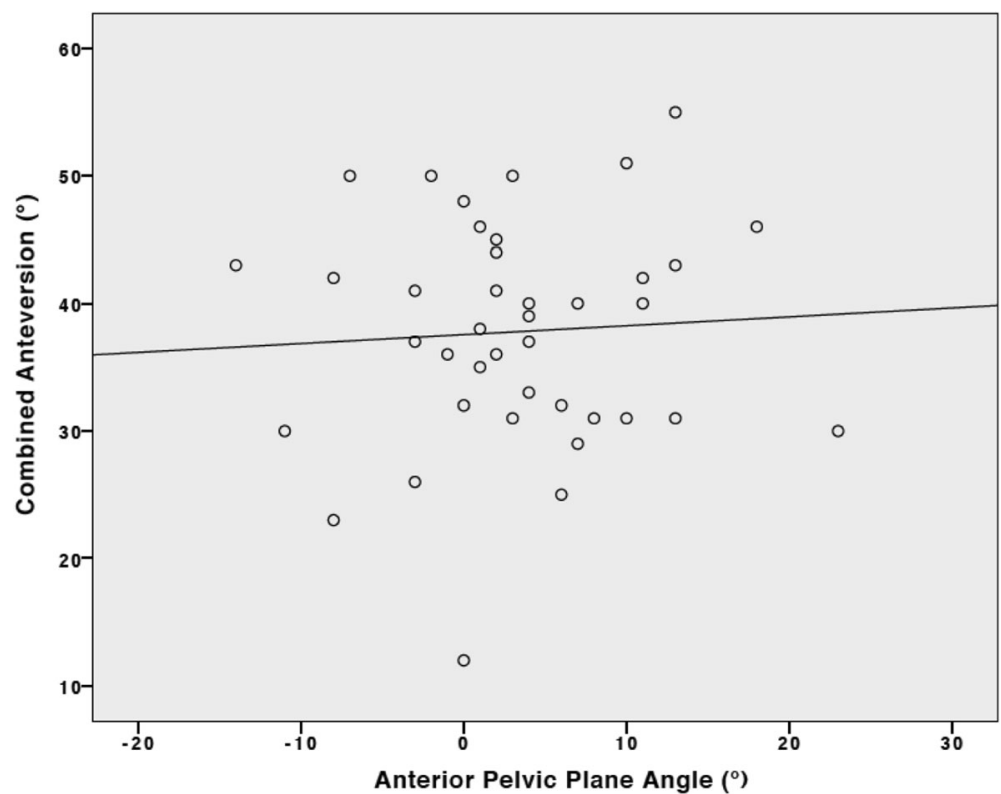

Fig. 4 Scatter plot between combined anteversion values measured with the cup-liner system and anterior pelvic plane values. Linear regression: $y=37.55+0.07 * x\left(R^{2}=0.004, P=0.71\right)$ 
In our series, the average postoperative stem antetorsion was $9^{\circ} \pm 10^{\circ}$ ranging from $23^{\circ}$ of retrotorsion to $31^{\circ}$ of antetorsion. These results are similar to those reported in previous studies. Dorr et al. [10] showed with the computer navigation a wide range of positions of uncemented femoral stems ranging from $17^{\circ}$ of retroversion to $28^{\circ}$ of antetorsion. Pierchon et al. [28] and Wines et al. [29] used a postoperative CT scans reporting a range from $30^{\circ}$ of retroversion to $45^{\circ}$ of antetorsion of both cemented and uncemented stems. The wide range of position of the stem in terms of torsion is related with the high variability in the geometry of the proximal femur. Because the stem should have the best fit in the bone its position is affected by the patient-specific femoral anatomy.

In our series, we reported an AI value within the targeted range in $92.5 \%$ of hips with an average value of $40^{\circ} \pm 6^{\circ}$. Although we reported 3 outliers, two of them were included in the group with varized stem. The range of $\mathrm{AI}$ from $30^{\circ}$ to $50^{\circ}$ suggested by Lewinnek et al. [1] was not related with the value of CCD angle of the stem. However, we believe that cup inclination should be congruent with the neck of the stem [15]. For this reason, we would suggest to use the femoral component to determine intraoperatively the proper AI. The definitive cup should be placed parallel with the horizontal line on the trial head in both axial and coronal plane. Thus, the surgeon is able to provide the coverage of the cup according with the CCD angle of the femoral stem. Our results demonstrated that the group with varized stem had AI values significantly higher than those reported in the group with standard stem $(P=0.007)$. In this respect, the two outliers of $56^{\circ}$ and $58^{\circ}$ respectively are acceptable values if associated with a varized stem. In the present study, we did not evaluate the effect of pelvic obliquity in the frontal plane on postoperative AI. However, because of the AI was determined by the CCD angle of the femoral stem, we believe that postoperative AI is not affected by pelvic obliquity.

About the second hypothesis, the combined anteversion value achieved with femur first technique was not affected by preoperative pelvic tilt. Because of the anteversion and inclination of the cup are strongly related with the patient-specific pelvic tilt and spine sagittal balance $[20,30]$, some authors suggested to use intraoperative navigation taking into account the preoperative pelvic tilt of the patient [31-34]. The "safe zone" suggested by Lewinnek et al. [1] was referred to bony landmarks assuming no tilt of the pelvis. However, in the most of patients the pelvic tilt is not neutral. For this reason, the navigation systems referring to anatomical bony landmarks need to consider the pelvic tilt and correct for their measurements to avoid inaccurate position of the cup. In the present technique, the surgeon did not need to consider the pelvic tilt. The patient was in the lateral position with the pelvis hold without change the patient-specific pelvic tilt and sagittal balance. After the femoral component was fixed in terms of antetorsion and CCD angle, the cup was positioned in a compliant fashion to the stem with the hip in neutral position at $0^{\circ}$ of flexion and abduction. Therefore, the cup was positioned according to the stem without tacking into account the preoperative pelvic tilt. In this respect, the cup was positioned according to the functional plane without changing the patient-specific pelvic tilt and spinal sagittal balance. Indeed, our results demonstrated that preoperative APP angle values did not correlate with the combined anteversion values after surgery.

During a THA procedure, the vast majority of surgeons place the prosthetic cup with the native acetabular anteversion and then place the stem achieving the best fit in the femur. Disadvantages of this approach include higher risk of hip dislocation and reduced ROMs due to a suboptimal combined anteversion $[35,36]$. To overcome these complications, some authors advocated the use of femur first technique to obtain an optimal combined anteversion. Dorr et al. [10] proposed to perform a cementless THA preparing the femur first with the estimation of antetorsion, and then implanting the cup with computer navigation. Authors recommended a mean combined anteversion of $35^{\circ}$ (range, $25^{\circ}$ to $45^{\circ}$ ). On the other hand, Unlu et al. [16] proposed a femur first procedure using the lesser trochanter as a landmark to estimate the femoral component antetorsion. They suggested to estimate intraoperatively the antetorsion of the femoral component by the formula: operative collo-trochanteric angle (defined as the angle between the collo-femoral axis and the lesser trochanter axis) minus $34^{\circ}$ (mean lesser trochanteric version). Finally, the position of the cup should be adjusted to provide a mean combined anteversion of $37^{\circ}$ (range, $25^{\circ}$ to $50^{\circ}$ ) by internal rotation without hip flexion with patient in lateral decubitus. Finally, Nakashima et al. [11] proposed to measure the stem antetorsion intraoperatively with a goniometer as the angle between the perpendicular to the axis of the tibia hold in vertical position and the neck of the stem. The cup was positioned with $20^{\circ}$ of anteversion by using the manufacturer's jig and then it was adjusted according the stem antetorsion to achieve the targeted combined anteversion.

Although previous studies evaluated the effectiveness of femur first technique to achieve a proper combined anteversion $[10,11,16]$, to our knowledge, this is the first study demonstrating that a non-computer-based femur first technique could be also used to obtain a proper inclination of the acetabular cup according with the CCD angle of the stem. Indeed, previous studies focused only to achieve a proper combined anteversion trough the femur first procedure. Moreover, we also 
demonstrated that the postoperative combined anteversion values achieved with this technique are not affected by preoperative APP angle values.

We are aware that the present study is affected by some limitations. First, this case series had no control cases who underwent primary THA with conventional surgical procedure. Therefore, we were not able to ascertain the superiority of femur first technique over a conventional procedure characterized by the implantation of the cup first according anatomical bony landmarks. Further studies should be performed to address this comparison. Second, all patients were operated by a single experienced surgeon. Therefore, further studies should be performed to compare in standardized fashion the outcomes achieved by junior and senior surgeons with this technique. However, in our current practice, this procedure is easily performed by all surgeons of the unit with reliable results. Third, the fixation of uncemented stem requires a stable press fit into the bone, and the implant must adapt to the variable femoral geometry. Therefore, to achieve the desired stem antetorsion could be difficult, and the present findings of antetorsion may not be referred to all commercial designs. For this reason, only two straight femoral stems were used. Moreover, the optimum range for combined anteversion is still controversial. In clinical setting, some authors proposed a range from $25^{\circ}$ to $50^{\circ}[10,16]$, whereas others suggested a range form $40^{\circ}$ to $60^{\circ}[11,37]$. The present technique may allow the surgeon to adjust the orientation of the cup basing on stem antetorsion in order to provide a reliable combined antiversion within the range between $25^{\circ}$ to $50^{\circ}$. Forth, we used a liner with $10^{\circ}$ elevated rim and the definitive combined anteversion was based on the anteversion of the cup-liner system and stem. However the position of the elevated rim was collected in a systematic fashion and the radiographic measurements of AA were corrected for. Fifth, we did not compare the intraoperative estimation of the stem antetorsion by the surgeon with the postoperative radiographic measurements. However, previous studies demonstrated the low accuracy of surgeon's estimation of the stem torsion. Dorr et al. [10] reported that intraoperative estimation by the surgeon had outliers between $6^{\circ}$ to $10^{\circ}$ and than $10^{\circ}$ in $23 \%$ of the hips respectively. Hirata et al. [38] demonstrated that the average value of error for the surgeon's intraoperative estimation of the stem anteversion was $7.3^{\circ}$ ranging from $11^{\circ}$ underestimation to $25^{\circ}$ overestimation. Moreover, the aim of the study was to evaluate the combined anteversion and AI values achieved with the femur first procedure.

\section{Conclusions}

The femur first technique is a non-computer-based procedure that allows the surgeon to achieve a combined anteversion ranging from $25^{\circ}$ to $50^{\circ}$ with a cup inclination ranging from $30^{\circ}$ to $50^{\circ}$. Moreover, the cup is positioned according to the functional plane of the patient regardless the preoperative pelvic tilt.

\section{Abbreviations}

AA: acetabular anteversion; Al: acetabular inclination; APP: anterior pelvic plane; CCD: caput-collum-diaphyseal; HHS: Harris Hip Score; ROM: range of motion; THA: total hip arthroplasty

\section{Acknowledgements}

We would thank the Livio Sciutto Foundation for Medical Research (www.fondazione.it). This is a Non-profit Social Organisation that recorded in its database the data of the patients included in the study, with the previous consent of the patients and respecting the current law on the privacy.

\section{Funding}

No external funding was received for this study. Livio Sciutto Foundation for Medical Research (www.fondazione.it) provided the payment for the publication fee.

\section{Availability of data and materials}

The datasets during and/or analysed during the current study available from the corresponding author on reasonable request.

\section{Authors' contributions}

GG, ML and VD designed the study protocol. EC and ADR collected the data. Analyses were planned by GG, ML, and UGL and were carried out by $M L$ and UGL. ML and VD wrote the initial draft of the manuscript, which was critically revised by VD and GG. All authors read and approved the final manuscript.

\section{Ethics approval and consent to participate}

The present study was approved by the Independent Ethics Committee of Humanitas Clinical and Research Centre (protocol n.1366). All enrolled patients gave their consent to participate in the study.

\section{Consent for publication}

Not applicable.

\section{Competing interests}

One author (GG) is a paid consultant for Zimmer Biomet. Two authors (ML and UGL) are members of the Editorial Board of BMC Musculoskeletal Disorders.

None of the other authors have any competing interests.

\section{Publisher's Note}

Springer Nature remains neutral with regard to jurisdictional claims in published maps and institutional affiliations.

\section{Author details}

${ }^{1}$ Hip Diseases and Joint Replacement Surgery Unit, Humanitas Clinical and Research Centre, Via Alessandro Manzoni 56, 20089 Rozzano, Milan, Italy. ${ }^{2}$ Humanitas University, Via Alessandro Manzoni 113, 20089 Rozzano, Milan, Italy. ${ }^{3}$ Department of Orthopaedic and Trauma Surgery, Campus Bio-Medico University, Via Alvaro del Portillo 200, 00128 Trigoria, Rome, Italy.

Received: 21 July 2016 Accepted: 24 July 2017

Published online: 01 August 2017

References

1. Lewinnek GE, Lewis JL, Tarr R, Compere CL, Zimmerman JR. Dislocations after total hip-replacement arthroplasties. J Bone Joint Surg Am. 1978;60(2):217-20.

2. Del Schutte H, Jr., Lipman AJ, Bannar SM, Livermore JT, Ilstrup D, Morrey BF. Effects of acetabular abduction on cup wear rates in total hip arthroplasty. J Arthroplast 1998;13(6):621-626.

3. Kennedy JG, Rogers WB, Soffe KE, Sullivan RJ, Griffen DG, Sheehan LJ. Effect of acetabular component orientation on recurrent dislocation, pelvic osteolysis, polyethylene wear, and component migration. J Arthroplast. 1998;13(5):530-4.

4. Kummer FJ, Shah S, Iyer S, DiCesare PE. The effect of acetabular cup orientations on limiting hip rotation. J Arthroplast. 1999;14(4):509-13. 
5. Biedermann R, Tonin A, Krismer M, Rachbauer F, Eibl G, Stockl B. Reducing the risk of dislocation after total hip arthroplasty: the effect of orientation of the acetabular component. J Bone Joint Surg Br. 2005;87(6):762-9.

6. Maynard MJ, Ranawac CS. Modern techniques of cemented total hip arthroplasty. Tech Orthop. 1991;6:17-25.

7. Widmer $\mathrm{KH}$, Zurfluh B. Compliant positioning of total hip components for optimal range of motion. J Orthop Res. 2004;22(4):815-21.

8. Yoshimine F. The safe-zones for combined cup and neck anteversions that fulfill the essential range of motion and their optimum combination in total hip replacements. J Biomech. 2006;39(7):1315-23.

9. Hisatome T, Doi H. Theoretically optimum position of the prosthesis in total hip arthroplasty to fulfill the severe range of motion criteria due to neck impingement. J Orthop Sci. 2011;16(2):229-37.

10. Dorr LD, Malik A, Dastane M, Wan Z. Combined anteversion technique for total hip arthroplasty. Clin Orthop Relat Res. 2009;467(1):119-27.

11. Nakashima Y, Hirata M, Akiyama M, Itokawa T, Yamamoto T, Motomura G, et al. Combined anteversion technique reduced the dislocation in cementless total hip arthroplasty. Int Orthop. 2014;38(1):27-32.

12. Sendtner $\mathrm{E}$, Muller M, Winkler R, Worner M, Grifka J, Renkawitz T. Femur first in hip arthroplasty-the concept of combined anteversion. Z Orthop Unfall. 2010;148(2):185-90.

13. Widmer $\mathrm{KH}$. Containment versus impingement: finding a compromise for cup placement in total hip arthroplasty. Int Orthop. 2007;31(Suppl 1):S29-33.

14. Widmer $\mathrm{KH}$, Majewski M. The impact of the CCD-angle on range of motion and cup positioning in total hip arthroplasty. Clin Biomech (Bristol, Avon). 2005;20(7):723-8

15. Sarmiento A, Ebramzadeh E, Gogan WJ, McKellop HA. Cup containment and orientation in cemented total hip arthroplasties. J Bone Joint Surg Br. 1990;72(6):996-1002

16. Unlu MC, Kesmezacar H, Kantarci F, Unlu B, Botanlioglu H. Intraoperative estimation of femoral anteversion in cementless total hip arthroplasty using the lesser trochanter. Arch Orthop Trauma Surg. 2011;131(9):1317-23.

17. Grappiolo G, Loppini M, Longo UG, Traverso F, Mazziotta G, Denaro V. Trabecular metal augments for the Management of Paprosky Type III defects without pelvic discontinuity. J Arthroplast. 2015;30(6):1024-9.

18. Harris WH. Advances in surgical technique for total hip replacement: without and with osteotomy of the greater trochanter. Clin Orthop Relat Res. 1980;146:188-204

19. Zini R, Longo UG, de Benedetto M, Loppini M, Carraro A, Maffulli N, et al. Arthroscopic management of primary synovial chondromatosis of the hip. Arthroscopy. 2013;29(3):420-6.

20. Lazennec JY, Brusson A, Rousseau MA. Hip-spine relations and sagittal balance clinical consequences. Eur Spine J. 2011;20(Suppl 5):686-98.

21. Lazennec JY, Brusson A, Dominique F, Rousseau MA, Pour AE. Offset and anteversion reconstruction after cemented and uncemented total hip arthroplasty: an evaluation with the low-dose EOS system comparing twoand three-dimensional imaging. Int Orthop. 2015;39(7):1259-67.

22. Lazennec JY, Rousseau MA, Rangel A, Gorin M, Belicourt C, Brusson A, et al. Pelvis and total hip arthroplasty acetabular component orientations in sitting and standing positions: measurements reproductibility with EOS imaging system versus conventional radiographies. Orthop Traumatol Surg Res. 2011;97(4):373-80.

23. Than P, Szuper K, Somoskeoy S, Warta V, Illes T. Geometrical values of the normal and arthritic hip and knee detected with the EOS imaging system. Int Orthop. 2012;36(6):1291-7.

24. Lazennec JY, Rousseau MA, Brusson A, Folinais D, Amel M, Clarke I, et al. Total hip prostheses in standing, sitting and squatting positions: an overview of our 8 years practice using the EOS imaging technology. Open Orthop J. 2015:9:26-44.

25. Boese CK, Dargel J, Oppermann J, Eysel P, Scheyerer MJ, Bredow J, et al. The femoral neck-shaft angle on plain radiographs: a systematic review. Skelet Radiol. 2016:45(1):19-28.

26. Sultan PG, Tan V, Lai M, Garino JP. Independent contribution of elevated-rim acetabular liner and femoral head size to the stability of total hip implants. Arthroplast. 2002;17(3):289-92.

27. Cobb TK, Morrey BF, Ilstrup DM. The elevated-rim acetabular liner in total hip arthroplasty: relationship to postoperative dislocation. J Bone Joint Surg Am. 1996;78(1):80-6.

28. Pierchon F, Pasquier G, Cotten A, Fontaine C, Clarisse J, Duquennoy A. Causes of dislocation of total hip arthroplasty. CT study of component alignment. J Bone Joint Surg Br. 1994;76(1):45-8.
29. Wines AP, McNicol D. Computed tomography measurement of the accuracy of component version in total hip arthroplasty. J Arthroplast. 2006:21(5):696-701.

30. Loppini M, Longo UG, Ragucci P, Trenti N, Balzarini L, Grappiolo G. Analysis of the pelvic functional orientation in the Sagittal plane: a radiographic study with EOS 2D/3D technology. J Arthroplast. 2017;32(3):1027-32.

31. Babisch JW, Layher F, Amiot LP. The rationale for tilt-adjusted acetabular cup navigation. J Bone Joint Surg Am. 2008;90(2):357-65.

32. DiGioia AM, Hafez MA, Jaramaz B, Levison TJ, Moody JE. Functional pelvic orientation measured from lateral standing and sitting radiographs. Clin Orthop Relat Res. 2006;453:272-6.

33. Lembeck $B$, Mueller $O$, Reize $P$, Wuelker N. Pelvic tilt makes acetabular cup navigation inaccurate. Acta Orthop. 2005;76(4):517-23.

34. Parratte S, Argenson JN. Validation and usefulness of a computer-assisted cup-positioning system in total hip arthroplasty. A prospective, randomized, controlled study. J Bone Joint Surg Am. 2007;89(3):494-9.

35. D'Lima DD, Chen PC, Colwell CW Jr. Optimizing acetabular component position to minimize impingement and reduce contact stress. J Bone Joint Surg Am. 2001;83(Suppl 2 Pt 2):87-91.

36. D'Lima DD, Urquhart AG, Buehler KO, Walker RH, Colwell CW Jr. The effect of the orientation of the acetabular and femoral components on the range of motion of the hip at different head-neck ratios. J Bone Joint Surg Am. 2000:82(3):315-21.

37. Jolles BM, Zangger P, Leyvraz PF. Factors predisposing to dislocation after primary total hip arthroplasty: a multivariate analysis. J Arthroplast. 2002;17(3):282-8.

38. Hirata M, Nakashima Y, Ohishi M, Hamai S, Hara D, Iwamoto Y. Surgeon error in performing intraoperative estimation of stem anteversion in cementless total hip arthroplasty. J Arthroplast. 2013;28(9):1648-53.

\section{Submit your next manuscript to BioMed Central and we will help you at every step:}

- We accept pre-submission inquiries

- Our selector tool helps you to find the most relevant journal

- We provide round the clock customer support

- Convenient online submission

- Thorough peer review

- Inclusion in PubMed and all major indexing services

- Maximum visibility for your research

Submit your manuscript at www.biomedcentral.com/submit
) Biomed Central 